\title{
OS 100 ANOS DA REVISTA DE MEDICINA DA FMUSP NA DIVULGAÇÃO DA PRODUÇÃO CIENTÍFICA
}

\author{
Suely Campos Cardoso ${ }^{1}$; Guilherme Yuiti Sikusawa ${ }^{2}$; Victor Eidi Koike Sakaguchi ${ }^{3}$; Gustavo \\ Rosa Gameiro ${ }^{4}$; Viktor Sinkunas ${ }^{5}$
}

*Faculdade de Medicina FMUSP, Universidade de São Paulo, São Paulo, SP, BR

Email: revistademedicina@fm.usp.br

\author{
Como citar: \\ CARDOSO, S. C; SIKUSAWA; G. Y.; SAKAGUCHI , V. E. K.; GAMEIRO, G. R.; SINKUNAS, V. Os 100 \\ anos da revista de medicina da FMUSP na divulgação da produção científica. In: ABEC MEETING, 1, 2017, \\ Curitiba. Anais... Curitiba: Associação Brasileira de Editores Científicos, 2017. p. 45-49. \\ http://dx.doi.org/10.21452/abecmeeting.2017.008
}

\begin{abstract}
Resumo: A Revista de Medicina do Departamento Científico (DC) da Faculdade de Medicina da USP (FMUSP), fundada em 1916, tem a missão de aproximar os acadêmicos da produção científica e aumentar a integração entre o corpo discente e docente. Editada, desde o início, por graduandos de medicina, é o periódico científico acadêmico mais antigo do mundo em circulação. Objetivos: Este trabalho pretende apresentar a evolução da Revista, de 1916 a 2017, com enfoque no seu centenário e nos atuais projetos de ampliação da presença no meio acadêmico nacional. Relato de experiência: Com lançamento em julho de 1916, a Revista de Medicina foi criada para divulgar a produção científica dos acadêmicos e docentes da FMUSP. Em 1981, ocorreu a indexação na base de dados LILACS, e, em 2000, na LATINDEX. A partir de 2013, entrou no Portal de Revistas da USP e em 2016, a Revista completou seu centenário com uma edição especial com 3 volumes e carta ao editor do laureado com prêmio Nobel em Medicina e Fisiologia: Dr. Bruce Beutler. Nesses 100 anos de publicações a Revista teve como maior desafio trabalhar dentro de um contexto de baixa produção científica na graduação. Em 2017, novos projetos, como parcerias com Congressos e os Núcleos Editorias Associados (NEAs), foram criados para ampliar a participação nacional da Revista. Resultados: Desde 2015, a Revista está entre os 161 periódicos do Portal de Revistas da USP como a mais acessada, com 770.303 acessos em 2016. Em 2017, ocorreram: a organização do projeto de fomento científico em NEAs, a passagem de 4 edições anuais para 6 a partir de 2018, as parcerias científicas com três dos maiores congressos universitários brasileiros (COMU, Braincoms e ABLAC) e o auxílio na organização científica do I Congresso dos Acadêmicos de Medicina do Espírito Santo. Conclusões: A Revista de Medicina tem desempenhado nos últimos 100 anos o papel de fomentar a escrita científica dos alunos da FMUSP, e, agora também de alavancar a produção científica dos acadêmicos de Medicina de todo o país.
\end{abstract}

Palavras-chave: Revista acadêmica. Publicações periódicas como assunto. Publicações periódicas.

Abstract: The Revista de Medicina of the Departamento Científico (DC) of Faculdade de Medicina da USP (FMUSP), founded in 1916, has the mission of bringing scholars closer to scientific production and increasing integration between the student body and the teaching staff. Edited from the outset by medical graduates, it is the oldest academic scientific journal in the world in circulation. Objectives: This study intends to present the evolution of the Journal, from 1916 to 2017, focusing on its centenary and current projects to increase its presence in the national academic environment. Experience: Launched in July 1916, Revista de Medicina was created to disseminate the scientific production of the academics and professors of FMUSP. In 1981, indexation occurred in the LILACS database, and in 2000 in LATINDEX. From 2013, entered the Portal USP de Revistas and in 2016, Journal completed its centennial with a special edition with 3 volumes and letter to the editor of the Nobel Prize in Medicine and Physiology: Dr. Bruce Beutler. In these 100 years of publications the journal had the greatest challenge to work within a context of low scientific production in undergraduate. In 2017, new projects, such as partnerships with Congresses and Associated Editorial Centers (AECs), were created to expand the Journal's national participation. Results: Since 2015, the Journal is among the 161 journals of the Portal USP de Revistas as the most accessed, with 770,303 accesses in 2016. In 2017, the following events occurred: the organization of the scientific development project in AECs, the passage of 4 annual issues for 6 as of 2018, the scientific partnerships with 3 of the largest Brazilian university congresses (COMU, Braincoms e ABLAC) and participation in the scientific organization of the I Congresso dos Acadêmicos de Medicina do Espírito Santo. Conclusions: Revista de Medicina has played in the last 100 years the role of fostering the scientific writing of FMUSP students, and now also leverage the scientific production of medical scholars from all over the country. Keywords: Academic scientific journal. Periodicals as topic. Periodicals. 
CARDOSO, S. C; SIKUSAWA; G. Y.; SAKAGUCHI , V. E. K.; GAMEIRO, G. R.; SINKUNAS, V. "Os 100 anos da revista de medicina da FMUSP na divulgação da produção científica"

\section{INTRODUÇÃO}

Apresentar uma Revista científica acadêmica que tem como tradição ser a revista acadêmica mais antiga do mundo em circulação. Esta revista tem uma proposta generalista, abordando sempre temas variados e de interesse de toda a comunidade. Um dos maiores desafios dos envolvidos nesta publicação é que seja uma referência nacional e internacional na difusão do conhecimento científico para os estudantes de Medicina e os profissionais da área de ciências da saúde. Tem como missão aproximar acadêmicos do mundo da produção científica, promover maior integração entre discentes e docentes, publicar artigos científicos que impulsionem o desenvolvimento da medicina e fomentar a escrita científica já na graduação. Sempre valorizando a Ética, Responsabilidade, Excelência e Cooperação. Este relato de experiência tem como finalidade apresentar aos estudantes e profissionais a trajetória de uma Revista acadêmica desde sua fundação até os dias atuais

\section{OBJETIVO}

Relatar a experiência do trabalho desenvolvido em uma revista científica acadêmica brasileira.

\section{RELATO DE EXPERIÊNCIA}

A Revista de Medicina foi fundada em 1916 por alunos do Centro Acadêmico da FMUSP, posteriormente foi criado o Departamento Científico em 1941 para gerenciar o periódico. Em 1981, ocorreu a indexação na base de dados LILACS, e, em 2000, na LATINDEX. Em 2012 e 2013 todos os volumes impressos da Revista desde 1916 foram digitalizados e a partir de 2013 foram integralmente disponibilizados de forma gratuita online no Portal de Revistas da USP. A partir desta iniciativa a divulgação da Revista só vem crescendo, sendo a Revista com mais download no Portal.

Nos últimos 3 anos (2015-2017), a Revista teve uma revitalização na sua gestão, com a designação de uma profissional contratada pela Universidade que ficasse disponível para trabalhar junto à Diretoria acadêmica da Revista. O Grupo passou a trabalhar em conjunto, procurando sempre melhorar a qualidade e divulgação da revista junto aos alunos, profissionais da saúde da FMUSP e de outras instituições acadêmicas do Brasil. Parte desse trabalho resultou na publicação de 3 edições comemorativas do centenário da Revista em 
2016, com participações de profissionais da área médica reconhecidos internacionalmente, como o laureado com prêmio Nobel de Medicina e Fisiologia: Dr. Bruce Beutler.

A Diretoria da Revista formada por acadêmicos vem trabalhando, nos últimos anos, com o objetivo de manter a qualidade da publicação bem como desenvolver outras iniciativas para que a Revista se torne referência junto a faculdades de Medicina do Brasil e se torne o primeiro periódico acadêmico com fator de impacto.

Para conseguir atingir a missão de aproximar os acadêmicos da produção científica a Revista de Medicina conta com uma disciplina optativa de Abordagem à Escrita Científica na FMUSP, na qual os alunos devem apresentar ao final um artigo de revisão em inglês com a orientação de um professor. Esse mesmo projeto está sendo direcionado para os Núcleos Editorias Associados de outras instituições para que o país tenha uma maior produção ainda na graduação.

\section{RESULTADOS}

Em março de 2015, a revista iniciou a submissão online e criação da Secretaria Executiva da Revista no Núcleo Editorial da FMUSP. No mesmo ano, início dos preparativos dos 3 números comemorativos do Centenário de criação e o periódico foi reconhecido e convidado a fazer parte dos arquivos do Cengage.

Em agosto de 2016, lançamento das edições comemorativas do Centenário em uma solenidade na Sala da Congregação da FMUSP. No mesmo ano continuou a ser o periódico mais acessado no Portal USP, como indicado no Gráfico 1. 
CARDOSO, S. C; SIKUSAWA; G. Y.; SAKAGUCHI , V. E. K.; GAMEIRO, G. R.; SINKUNAS, V.

"Os 100 anos da revista de medicina da FMUSP na divulgação da produção científica"

Gráfico 1. Estatísticas do acesso a Revista de Medicina no Portal de Revistas da USP.

Total de Downloads por Revista em 2016 no Portal USP

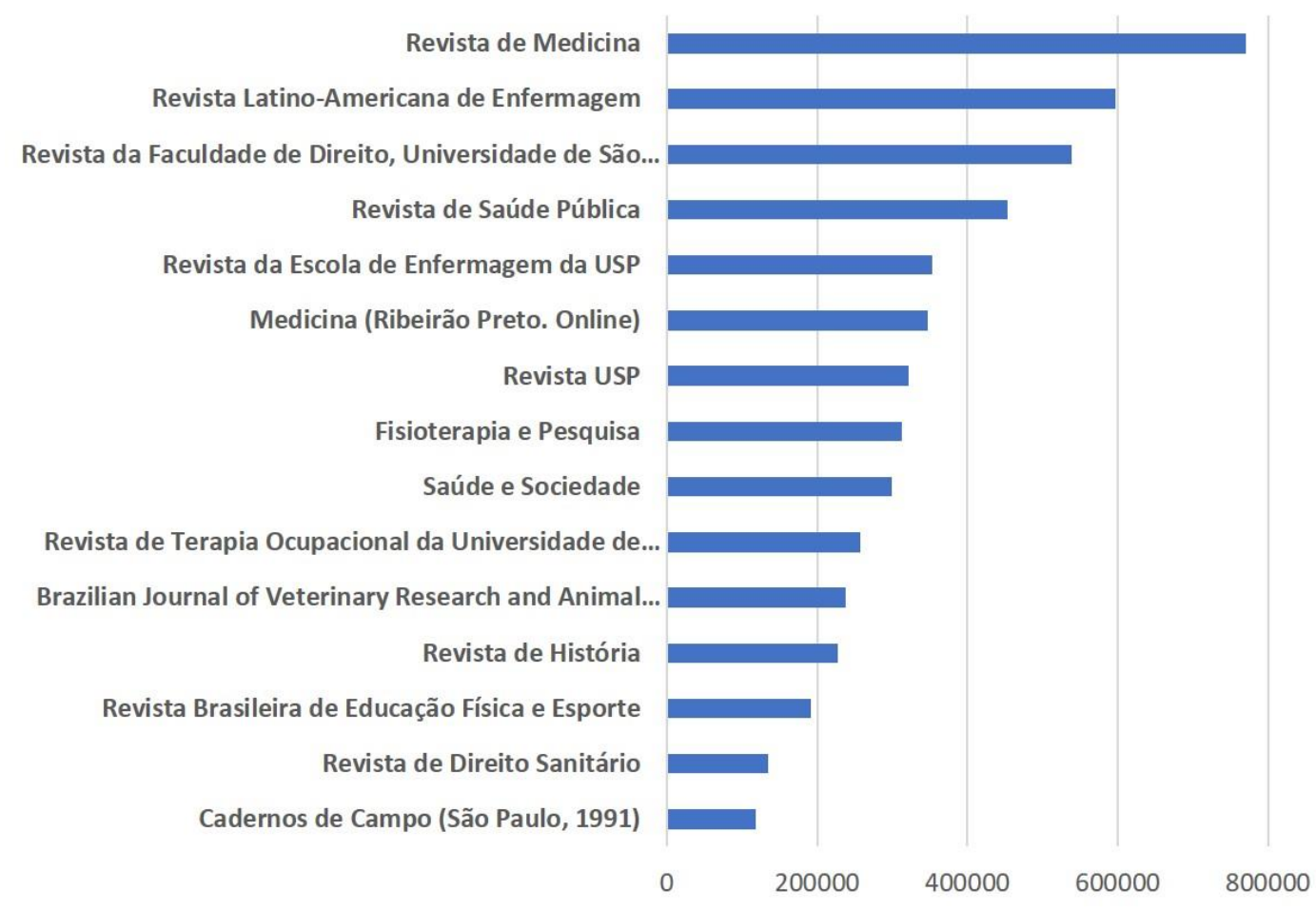

De janeiro a setembro de 2017 a Revista teve um aumento de 67 para 242 revisores especialistas de diversas instituições de ensino e pesquisa do Brasil. No mesmo período foi acessada em 62 países. Até o momento no ano foram publicados 31 artigos, 4 editoriais e 4 cartas ao editor, com 12 artigos aceitos para serem publicados próximo 4 e 40 artigos estão sendo avaliados pelos revisores.

A Tabela 1 mostra os 7 artigos publicados na Revista com o maior número de acessos/downloads até setembro de 2017, vale ressaltar que estas estatísticas estão sendo computadas desde junho de 2013, quando foram submetidas no Portal de Revistas da USP.

Tabela 1: Os 7 artigos publicados na revista maior visibilidade no Portal da USP, desde junho de 2013.

\begin{tabular}{clc}
\hline Posição & \multicolumn{1}{c}{ Artigo } & Visualizações \\
1 & Crescimento e desenvolvimento puberal na adolescência & 96.615 \\
2 & O escore de risco de Framingham para doenças cardiovasculares & 71.448 \\
3 & Síndrome metabólica: diagnóstico e tratamento & 66.770 \\
4 & Hérnias em geral: revisão didática & 53.435 \\
5 & Câncer de colo uterino - fatores de risco, prevenção, diagnóstico e tratamento & 39.580 \\
6 & Trauma urológico & 36.948 \\
7 & Escroto agudo & 36.620 \\
\hline
\end{tabular}

Resumos do ABEC MEETING, v. 1, n. 1, Curitiba (PR), 2017. 


\section{CONCLUSÕES}

O número expressivo de submissões acadêmicas em 2017 corrobora com a expectativa de que o grupo está colaborando com a publicação e produção científica nacional. A manutenção do trabalho da Diretoria, Secretaria executiva e dos Núcleos Editorias Associados objetivando novas indexações poderá, portanto, transformar a Revista de Medicina no primeiro periódico acadêmico brasileiro com impacto científico internacional.

\section{REFERÊNCIAS}

CARDOSO, Suely Campos; SANCHEZ, Fabiola Rizzo; CRESTANA, Maria Fazanelli. Trajetória dos 90 anos de publicação da Revista de Medicina. Revista de Medicina, v. 85, n. 4, p. 168-173, 2006.

CARDOSO, S. C.; SANCHEZ, F. R.; FREITAS, T. Q.; POLHO, G. B.; GAMEIRO, G. R. Revista de Medicina e sua trajetória: 1916 a 2016. In: XV ENCONTRO NACIONAL DE EDITORES CIENTÍFICOS, 2, 2015, Florianópolis. Anais... Florianópolis: Associação Brasileira de Editores Científicos, p. 1-6. Disponível em: <http://ocs.abecbrasil.org.br/index.php/ENEC/enec/paper/view/100>. Acesso em: 10 set. 2017.

GAMEIRO, G. R. Experiência da Revista de Medicina: estratégias de divulgação científica por meio de mídias digitais. In: FÓRUM DE PERIÓDICOS CIENTÍFICOS, 2015, São Paulo. Anais... São Paulo: Universidade Federal de São Paulo. Disponível em: <http://pt.slideshare.net/sibiusp/gustavo-rosa-gameiro-experincia-da-revista-de-medicina>. Acesso em: 10 set. 2017. 